\title{
CARCINOMA RENAL DE LOS CONDUCTOS COLECTORES DE BAJO GRADO. PRESENTACIÓN DE UN CASO Y REVISIÓN DE LA LITERATURA
}

\author{
P. SAN MIGUEL FRAILE, J.E. DOS SANTOS, C. DELGADO, E. ZUNGRI TELO, \\ C. ÁLVAREZ, B. IGLESIAS
}

Servicio de Patología. *Servicio de Urología. **Servicio de Radiología. Centro Médico POVISA. Vigo (Pontevedra).

Actas Urol Esp. 28 (6): 478-483, 2004

\section{RESUMEN}

CARCINOMA RENAL DE LOS CONDUCTOS COLECTORES DE BAJO GRADO. PRESENTACIÓN DE UN CASO Y REVISIÓN DE LA LITERATURA

El carcinoma renal de los conductos colectores es una variante poco frecuente de carcinoma renal. Típicamente su comportamiento es más agresivo que el de otras formas de carcinoma de células renales, siendo habitualmente diagnosticado en estadios avanzados. Presentamos un caso de carcinoma renal de los conductos colectores de bajo grado. Un varón de 57 años fue admitido en nuestro hospital con clínica de cólico nefrítico. En el TAC se observó una masa en el riñón derecho y se le realizó una nefrectomía parcial. El diagnóstico histológico fue carcinoma renal de los conductos colectores de bajo grado. Es una entidad nueva, de comportamiento desconocido y aparentemente menos agresivo que el carcinoma de los conductos colectores clásico. Describimos las características clínicas, radiológicas, histológicas e inmunohistoquímicas de este tumor y revisamos la literatura.

PALABRAS CLAVES: Carcinoma de los conductos colectores. Bellini. Bajo grado. Riñón.

\section{ABSTRACT \\ LOW GRADE COLLECTING DUCT CARCINOMA OF THE KIDNEY. PRESENTATION OF ONE CASE AND LITERATURE REVISION}

Collecting duct renal cell carcinoma is an uncommon variant of renal carcinoma. Typically its behaviour is more aggressive than other forms of renal carcinoma and usually it is diagnosed at advanced stages. A 57-year-old man visited our hospital by right lumbar pain. Abdominal CT showed a enhanced mass on the right kidney. A right partial nephrectomy was done. Histological examination of the surgical specimen showed a low grade collecting duct carcinoma of the kidney. This is a new entity, with unknown behaviour but seems to be less aggressive than classical collecting duct carcinoma of the kidney. The clinical, radiological, pathological and immunohistochemical characteristics of this tumor are reported, and the literature is reviewed.

KEY WORDS: Collecting duct carcinoma. Bellini. Low grade. Kidney. 
$\mathrm{E}$ 1 carcinoma renal de los conductos colectores (CRCC) es una variante infrecuente de tumor renal $(1-3 \%)^{1,2}$. Estudios previos han sugerido su origen en los conductos colectores, basándose en la localización predominantemente medular del tumor, la similitud histológica con los túbulos colectores renales, y la presencia de atipia y/o hiperplasia en los ductos colectores próximos a la neoplasia $^{1,3}$. Sin embargo, la expresión de citoqueratinas de alto peso molecular parece ser el hallazgo histológico más evidente que confirma este origen ${ }^{4}$.

La infrecuencia e inusual apariencia histológica de este tumor, puede hacer que plantee diagnóstico diferencial con neoplasias de otro origen, sobre todo cuando es la metástasis la forma inicial de presentación ${ }^{3}$. Presentamos un caso de CRCC de bajo grado localizado, en el momento de diagnóstico, que fue tratado con nefrectomía parcial. Analizamos las características clínicas, histológicas e inmunohistoquimicas de esta variante tumoral infrecuente y revisamos la literatura.

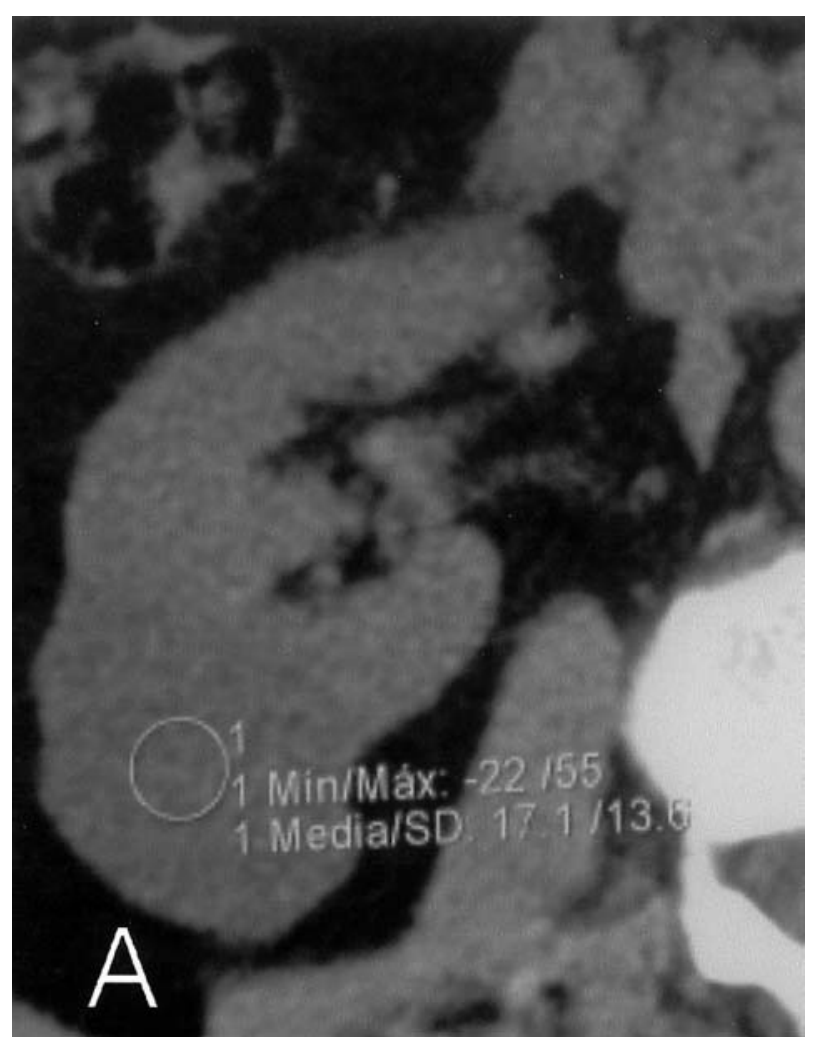

\section{CASO CLÍNICO}

Varón de 57 años de edad (№ H $^{\text {a }}$ 3877389) con antecedentes de resección de sigma por adenocarcinoma intestinal hace 6 años, que fue remitido a nuestro hospital con el diagnóstico de una masa renal derecha descubierta en el transcurso de un estudio radiológico por cólicos nefríticos de repetición. La exploración física no mostró hallazgos de interés. En las determinaciones analíticas sanguíneas todos los parámetros estaban dentro de la normalidad excepto la glucosa (122 mgr/dl), ácido úrico (7,70 mgr/dl) y colesterol (275 mgr/dl). Los estudios radiológicos pusieron de manifiesto por ecografia y Tomografia Axial Computerizada (TAC) una lesión en la región interpolar del riñón derecho. Ecográficamente era una lesión isoecoica con el resto del parénquima y moderadamente exofitica de aproximadamente $5 \mathrm{cms}$ de diámetro. En el TAC se visualizó una lesión hipodensa de 4,5 cms de diámetro céfalo-caudal de contorno discretamente lobulado y crecimiento exofítico y que se realzaba ligeramente tras la administración de contraste intravenoso (Fig. 1). Se recomendó rea-

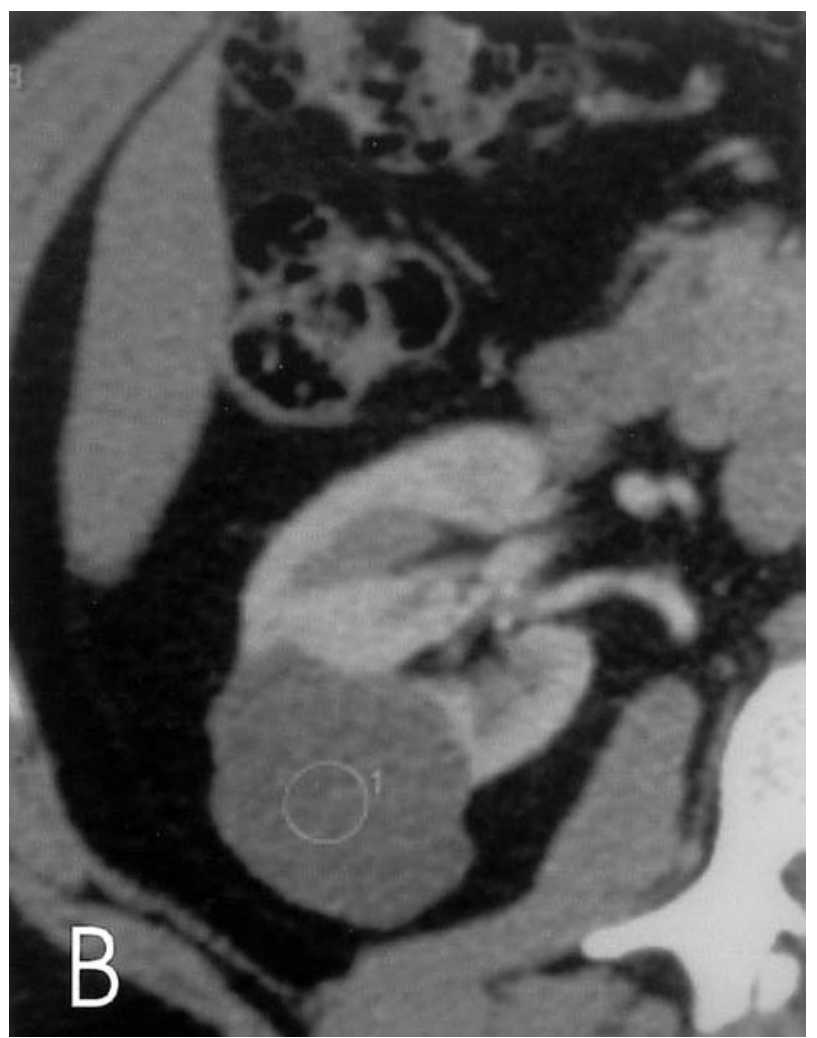

FIGURA 1. Imagen de TAC sin (A) y con (B) contraste intravenoso. El tumor se comporta como una lesión sólida que se realza levemente tras la administración de contraste. Intravenoso. 
lizar una resonancia magnética, en la que se observó una masa compleja en región interpolar del riñón derecho con crecimiento exofitico que medía aproximadamente 4,3 x 4,1 cms de diámetro, definiéndose en su interior múltiples imágenes quísticas de pequeño tamaño separadas por finos septos, que se realzaba ligeramente después de la administración de contraste. No se identificaron adenopatías patológicas retroperitoneales, metástasis en glándulas suprarrenales ni trombos en vena renal o cava inferior. Con las técnicas de imagen las principales opciones diagnósticas fueron nefroma quístico multilocular o carcinoma renal quístico. Se realizó nefrectomía parcial derecha por incisión lumbar con comprobación histológica pre-operatoria de los bordes quirúrgicos de resección: bordes quirúrgicos libres.

Macroscópicamente se recibió una pieza de nefrectomía parcial que pesaba 109 grs y medía $6,5 \times 4,5 \times 4,5 \mathrm{cms}$. La mayor parte de la pieza estaba ocupada por una tumoración excéntrica de límites bien definidos que no parecía infiltrar macroscópicamente la grasa ni parénquima renal adyacente. La tumoración era de coloración grisácea, aspecto microquístico y tenía un diámetro mayor de $5 \mathrm{cms}$ (Fig. 2). No contactaba con los bordes quirúrgicos de resección. El estudio histológico mostró una tumoración conformada por múltiples ductos de diverso tamaño que estaban tapizados por un epitelio cúbico o plano adoptando en algunas ocasiones una morfología en "tachuela". Las células mostraban un ligero aumento de la

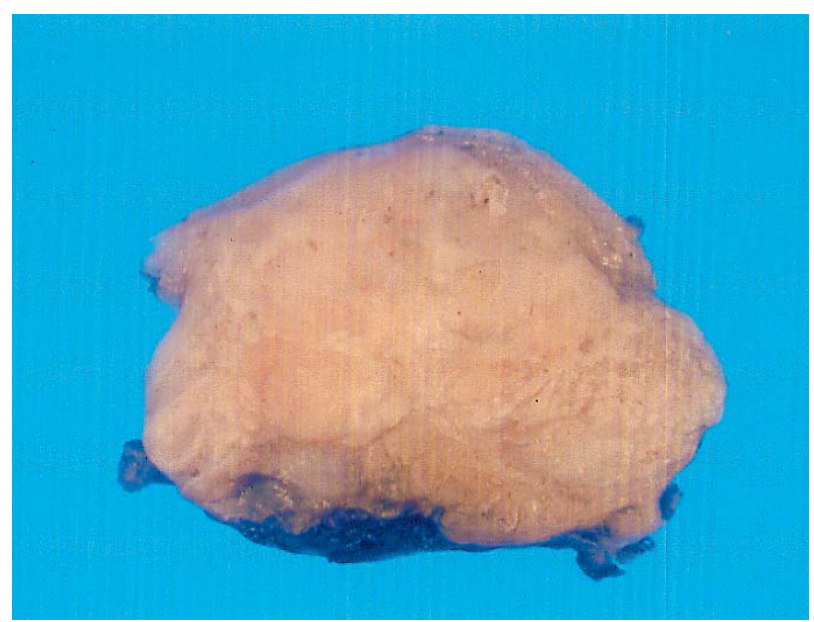

FIGURA 2. Pieza de nefrectomía parcial, ocupada por una tumoración excéntrica de limites bien definidos. relación núcleo/citoplasma y nucleolo llamativo, siendo las mitosis muy ocasionales. Los ductos eran de diversos tamaños y adoptaban en algunas ocasiones un patrón microquístico. Entre los ductos se apreciaba un estroma con marcada desmoplasia (Fig. 3). No se observaban áreas de necrosis ni hemorragia. En el parénquima renal circundante se observa en los túbulos colectores áreas focales de hiperplasia y displasia que no contactaban con el borde quirúrgico de resección (Fig. 4). Se realizó estudio con técnicas de inmunohistoquímica mostrando las células tumorales positividad intensa para queratinas (AE 1-AE3), queratinas de alto peso molecular (CK7) (Fig. 5) y

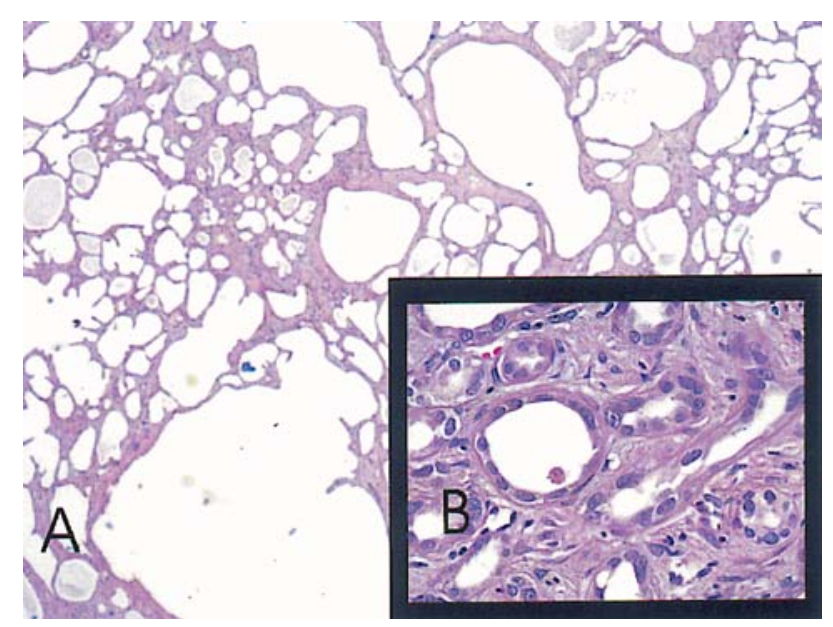

FIGURA 3. Proliferación tubular con estroma desmoplásico (A: Hematoxilina- eosina $x$ 150) (B: Hematoxilina-eosina $x$ 200).

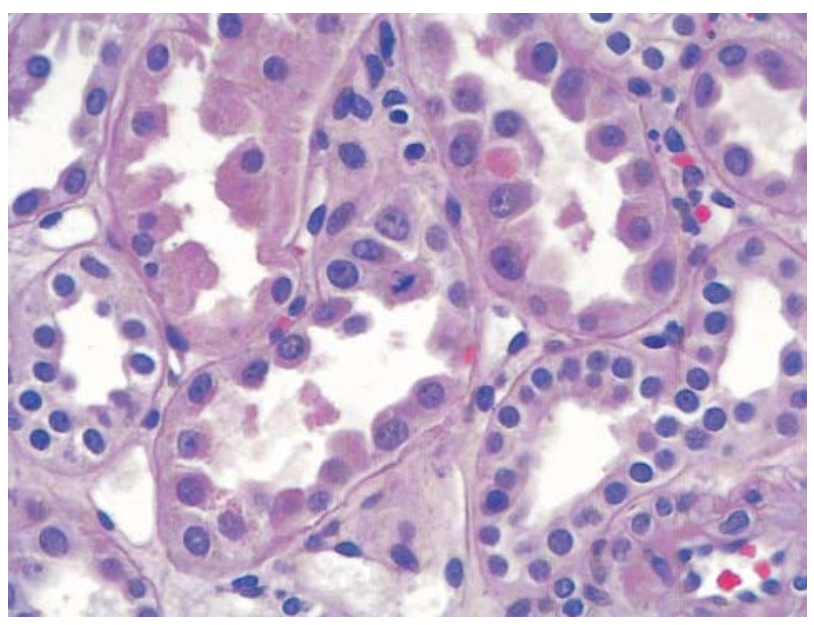

FIGURA 4. Hiperplasia atípica en conductos colectores adyacentes a la neoplasia (Hematoxilina-eosina $x$ 200). 

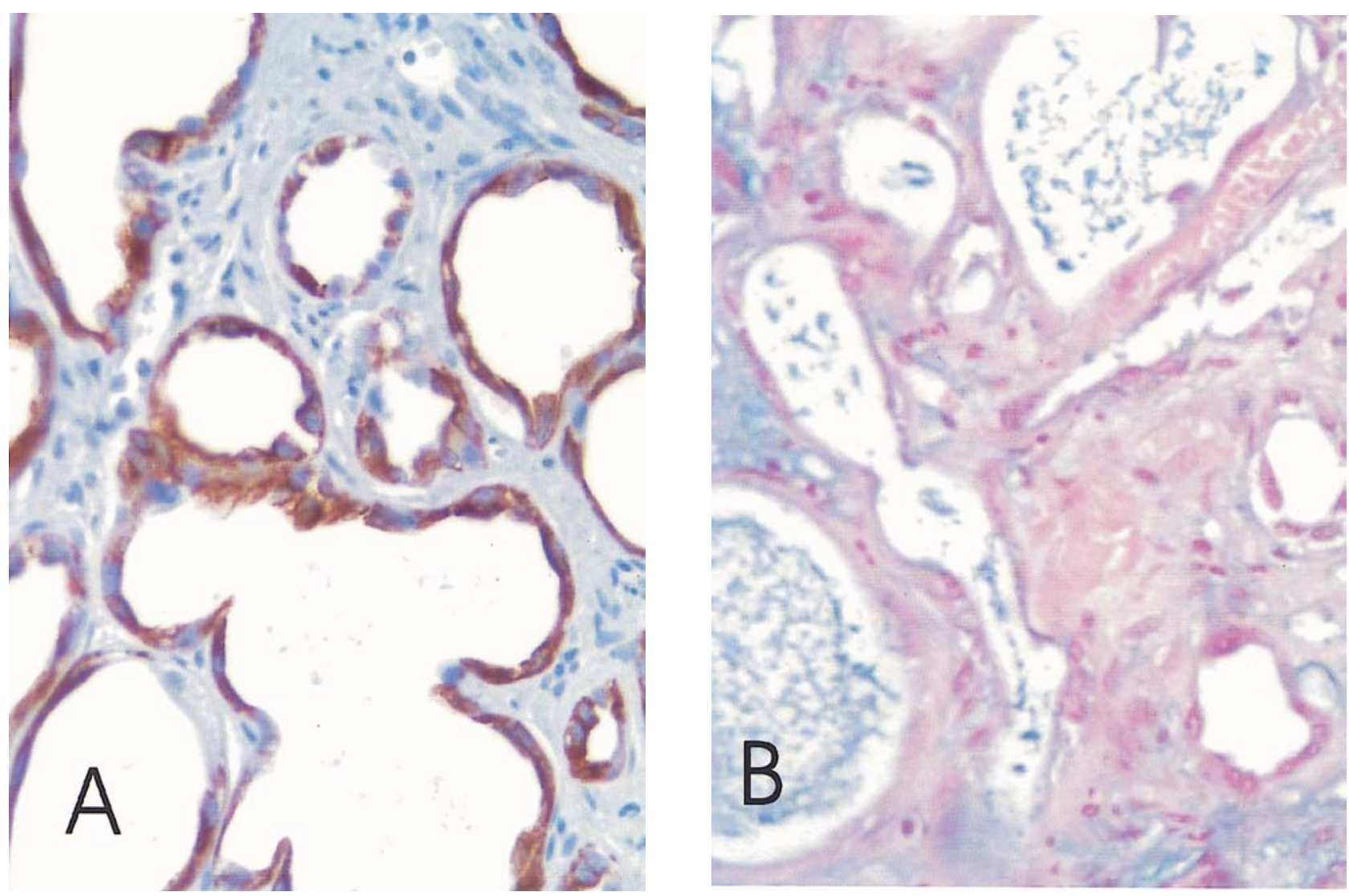

FIGURA 5. A) Positividad difusa para citoqueratina de alto peso molecular (CK $7 \times 200)$. B) Positividad difusa con la técnica de Azul alcián, demostrando producción de mucina (Azul Alcián x 200).

vimentina; positividad débil para antígeno de membrana epitelial (EMA) y queratinas de alto peso molecular (34b E12) y negatividad para antígeno carcino embrionario (CEA) y Ulex europeus. El diagnóstico definitivo fue carcinoma de los conductos colectores de $5 \mathrm{cms}$ de diámetro mayor que no infiltraba grasa y respetaba bordes quirúrgicos de resección. Estadio pTlb y grado II de Furhman. En la actualidad, con un seguimiento de 4 meses el paciente se encuentra asintomático y libre de tumor.

\section{DISCUSIÓN}

El carcinoma renal de los conductos colectores (CRCC) constituye una variante poco frecuente de tumor renal (1-3\%) $)^{1,2}$, que muestra características clínicas, histológicas e inmunohistoquímicas (IHQ) diferentes del carcinoma de células renales (CCR). Este tumor fue descrito por primera vez por Pierre Masson ${ }^{5}$ en 1970 y lo denominó "epitelioma Belliniano" por su similitud his- tológica con los conductos de Bellini. En 1976, Mancilla Jiménez y cols. ${ }^{6}$ describieron una variante de carcinoma renal con patrón papilar y comentaron su posible origen en los conductos colectores, por los cambios hiperplásicos y atípicos que se observaban en los túbulos colectores adyacentes a la neoplasia. Pero fue en 1986 cuando Fleming y Lewi ${ }^{1}$, quienes describieron las características clínicas e histológicas de esta entidad y establecieron el origen de este tumor en los conductos de Bellini, basándose fundamentalmente en sus características clínicas, localización medular, la similitud histológica e inmunohistoquímica con el epitelio de los conductos colectores y la atipia y/o hiperplasia de los conductos colectores adyacentes a la neoplasia. Desde entonces no son más de 100 los casos de CRCC publicados en la literatura internacional y 13 los casos descritos en revistas nacionales ${ }^{7-12}$.

Clínicamente, su comportamiento más agresivo y su peor pronóstico ${ }^{1,3,13,14}$, así como su 
localización predominantemente medular ${ }^{13}$ y su presentación en edades más tempranas ${ }^{1}$ lo diferencian claramente del carcinoma de células renales.

La mayoría de los casos en el momento del diagnóstico se presentan en un estadio clínico avanzado con invasión de la cápsula renal o metástasis a distancia ${ }^{14}$. Los principales asentamientos de las metástasis son: ganglios linfáticos locales $^{15}$ o a distancia ${ }^{2,3,13,15}$, hueso, ${ }^{4,13,14}$, hígado ${ }^{14}$ y pulmón ${ }^{4,13,14}$.

Radiológicamente suelen ser tumores de localización central con preservación del contorno renal, hipovasculares y mínimo realce con contraste $^{8,16}$. En nuestro caso, se trataba de un tumor exofitico y polilobulado que afectaba a médula y corteza renal y distorsionaba el parénquima; fenómeno no habitual en el CRCC. Sin embargo en la resonancia magnética sí que mostraba características peculiares como es el patrón microquístico del tumor, lo que hizo que se sugiera como principal opción diagnóstica un nefroma quístico o un carcinoma renal quístico y en el TAC se apreció mínimo realce con contraste, que es infrecuente en el hipernefroma.

Macroscópicamente, suelen ser tumores de gran tamaño, localización medular y que infiltran secundariamente la corteza renal. Es frecuente el aspecto microquístico de la neoplasia, ya que está formado por estructuras tubulares dilatadas que le dan una morfología típica en "esponja". Es infrecuente la necrosis y hemorragia, a pesar de que suelen ser tumores de alto grado histológico y mal pronóstico.

Histológicamente es un tumor constituido por una proliferación tubular o tubulopapilar que crece en el seno de un estroma desmoplásico con moderado infiltrado inflamatorio acompañante y alto grado nuclear ${ }^{3}$. Este patrón histológico difiere del adenocarcinoma mucinoso primario de pelvis renal que presenta un patrón histológico similar a los adenocarcinomas de origen gástrico o intestinal.

Sin embargo, a pesar de tener unas características macro y microscópicas bien definidas, esta entidad a veces es difícil de diagnosticar sobre todo debido a su baja incidencia. El patrón inmunohistoquímico puede contribuir a realizar el diagnóstico ya que suele mostrar positividad para queratinas, especialmente las de alto peso molecular y suelen ser productores de mucina, lo que determina su origen en los conductos colectores distales $2,3,17,18$. Por otra parte, también está descrita la positividad con grado de expresión variable para el Ulex europeus, aglutininas, lecitinas, antígeno de membrana epitelial, lisozima y vimentina ${ }^{2,3,18}$. El patrón inmunohistoquímico del CRCC, así como las características citogenéticas son más semejantes al carcinoma transicional de pelvis renal que al carcinoma de células renales ${ }^{18}$.

Recientemente, se han descrito dos variantes histológicas de esta entidad: la variante sarcomatoide $^{17}$ y el CRCC de bajo grado ${ }^{19}$. El caso aquí descrito corresponde a esta última variante. Nuestro caso se trata de un tumor con patrón tubuloquístico, productor de mucina, con desmoplasia e hiperplasia atípica en conductos colectores próximos a la neoplasia cumpliendo criterios histológicos propios del CRCC. Sin embargo la atipia citológica no es tan llamativa como se describe habitualmente en un CRCC convencional. En 1997 McLennan y cols. ${ }^{19}$ describen 13 casos de CRCC de bajo grado y comentan las características clínicas, histológicas e inmunohistoquímicas de este tumor. En esta serie todos los tumores eran de grado histológico 1 ó 2 de Furhman. El estadio clínico de los tumores fue T1 en 7 casos (55\%), T2 en 4 (39\%) y T3b en 2 (15\%). Dos pacientes fallecieron por la neoplasia y en dos casos fue un hallazgo de autopsia. Los pacientes restantes estaban vivos y libres de enfermedad después de un seguimiento clínico medio de 62 meses.

El pronóstico del CRCC de bajo grado parece más favorable que la forma convencional ${ }^{14}$, no obstante estos resultados deben de ser tomados con cautela ya que el número de casos descritos en la literatura es muy bajo y dos pacientes con CRCC de bajo grado (15\%) fallecieron por enfermedad metastásica o múltiples recurrencias ${ }^{19}$.

Con respecto al tratamiento del CRCC no hay uno establecido, habiéndose realizado en los casos descritos nefrectomía radical ${ }^{3,13,19}$, y menos frecuentemente parcial ${ }^{20}$, como es nuestro caso. Carter ${ }^{15}$ y Milowsky ${ }^{18}$ sugieren el tratamiento con agentes quimioterápicos o incluso inmunoterapia (interferón alfa, interleukina 2 o 
combinación de ambos) basándose en la gran similitud inmunohistoquímica y citogenética con el carcinoma transicional. Esta similitud es debida al mismo origen embriológico en el conducto de Wolff de los túbulos colectores, uréter, pelvis y cálices renales y por lo tanto del carcinoma transicional y $\mathrm{CRCC}^{3,15,18}$.

En resumen aportamos un nuevo caso de CRCC de bajo grado, describimos las características clínicas, radiológicas, histológicas e inmunohistoquímicas de este tumor y comentamos la necesidad de diferenciarlo del CRCC convencional por su menor grado de atipia nuclear y aparentemente mejor pronóstico, aunque estos resultados deben ser confirmados mediante estudios en series más amplias.

\section{CONCLUSIÓN}

El CRCC de bajo grado es un tumor infrecuente, que debemos diferenciar histológicamente del CRCC convencional por su aparente mejor pronóstico. Estos resultados deben ser confirmados mediante estudios en series más amplias, debido al escaso número de casos descritos hasta el momento en la literatura.

\section{REFERENCIAS}

1. FLEMING S, LEWI HJ.: Collecting duct carcinoma of the kidney. Histopathology 1986; 10: 1131-1141.

2. DIMOPOULOS MA, LOGOTHETIS CJ, MARKOWITZ A, SELla A, AMATO R, RO J.: Collecting duct carcinoma of the kidney. Br J Urol 1993; 71: 388-391.

3. KENNEDY S, MERINO M, LINEHAN WM, ROBERTS JR, ROBERTSON CN, NEUMANN RD.: Collecting duct carcinoma of the kidney. Hum Pathol 1990; 21: 449-456.

4. FLEMING S, SYMES CE.: The distribution of cytokeratin antigens in the kidney and in renal tumours. Histopathology 1987; 11: 157-170.

5. MASSON P.: Tumeurs humines 1955, in Kobernick $\mathrm{S}$ : human tumors, histology, diagnosis and technique, (ed 2). Detroit MI, Wayne State University Press, 1970, chap 5.

6. BAER SC, RO JY, ORDÓÑEZ NG, MIESE RL, LOOSE JH, GRIGNON DG, AYALA AG.: Sarcomatoid collecting duct carcinoma: a clinicopathologic and immunohistochemical study of five cases. Hum Pathol 1993; 24 (9): 1017-1022.

7. PORTILLO JA, VAL F, MARTÍN B y cols.: Carcinoma de los conductos de Bellini. Actas Urol Esp 1993; 17: 598-602.

8. ABAD C, GARCÍA D, BELLAS MR y cols.: Carcinoma de los conductos colectores de Bellini. Actas Urol Esp 1994; 18: 761-763.
9. MONSERRAT V, LÓPEZ E, PÉREZ F y cols.: Carcinoma de ductos de Bellini a propósito de un caso. Arch Esp Urol 1997; 50: 798-799.

10. GARMENDIA JC, ARRINDA JM, HERNÁNDEZ Y y cols.: Carcinoma del conducto colector de Bellini, aportación de dos nuevos casos. Actas Urol Esp 1995; 19: 721-723.

11. PEREIRA JG, ATECA R, GUTIÉRREZ JM y cols.: Asociación sincrónica de carcinoma de los conductos colectores de Bellini y carcinoma transicional piélico. Actas Urol Esp 1997; 21: 990-996.

12. DE DIEGO E, PASCUAL C, GUTIÉRREZ JL, MARTÍN B, HERNÁNDEZ R, PORTILLO JA, CORREAS MA, DEL VALE JI, ROCA A, VILLANUEVA A, RADO MA, HERNÁNDEZ A.: Carcinoma de Bellini. Nuestra experiencia. Arch Esp Urol 2000; 53 (7): 611-616.

13. CHAO D, ZISMAN A, PANTUCK AJ, GITLITZ BJ, FREEDLAND SJ, SAID JW, FIGLIN RA, BELLDEGRUN AS.: Collecting duct renal cell carcinoma: clinical study of a rare tumour. J Urol 2002; 167: 71-74.

14. MÉJEAN A, ROUPR T M, LAROUSSERIE F, HOPIRTEAN V, THIOUNN N, DUFOUR B.: Is there a place for radical nephrectomy in the presence of metastatic collecting duct (Bellini) carcinoma. $J$ Urol 2003; 169 (4): 1287-1290.

15. CARTER MD, THA S, MCLOUUGLIN MG, OWEN DA.: Collecting duct carcinoma of the kidney: a case report and review of the literature. $J$ Urol 1992; 147: 1096-1098.

16. FUKUYA T, HONDA H, GOTO K, ONO M, MATSUURA T, KANEKO et al. Computed tomographic findings of Bellini duct carcinoma of the kidney. $J$ Comput Assist Tomogr 1996; 20: 399-403.

17. BAER SC, RO JY, ORDÓÑEZ NG, MAIESE RL, LOOSE JH, GRIGNON DG et al.: Sarcomatoid collecting duct carcinoma: a clinicopathologic and immunohistochemicl study of five cases. Hum Pathol 1993; 24: 1017-1022.

18. MCLENNAN GT, FARROW GM, BOSTWICK DG.: Low grade collecting duct carcinoma of the kidney: report of 13 cases of low-grade mucinous tubulocystic renal carcinoma of possible collecting duct origin. Urology 1997; 50: 679-684.

19. MILOWSKY MI, ROSMARIN A, TICKOO SK, PAPANICOLAU N, NANUS DM.: Active chemotherapy for collecting duct carcinoma of the kidney. A case report and review of the literature. Cancer 2002; 94: 111-116.

20. YASUNAGA Y, NISHIMURA K, TAKATERA $H$, FUJIIOKA H, TSUJIMOTO M.: Bellini duct carcinoma treated with partial nephrectomy: a case report. Acta Urologica Japonica 1994; 40 (12): 1103-1107.

Dra. P. San Miguel Fraile

C/ Santa Baia, 22 - portal 1, $3^{\circ} \mathrm{U}$

36208 Vigo (Pontevedra)

(Trabajo recibido el 17 octubre de 2003) 